\title{
Strong Convergence Results for Hierarchical Circularly Iterative Method about Hierarchical Circularly Optimization
}

\author{
Hongbo Liu \\ School of Science, Southwest University of Science and Technology, Mianyang, China \\ Email: liuhongbo@swust.edu.cn
}

Received June 20, 2013; revised July 22, 2013; accepted August 26, 2013

Copyright (C) 2013 Hongbo Liu. This is an open access article distributed under the Creative Commons Attribution License, which permits unrestricted use, distribution, and reproduction in any medium, provided the original work is properly cited.

\begin{abstract}
An hierarchical circularly iterative method is introduced for solving a system of variational circularly inequalities with set of fixed points of strongly quasi-nonexpansive mapping problems in this paper. Under some suitable conditions, strong convergence results for the hierarchical circularly iterative sequence are proved in the setting of Hilbert spaces. Our scheme can be regarded as a more general variant of the algorithm proposed by Maingé.
\end{abstract}

Keywords: Hierarchical Optimization Problems; Circularly Variational Inequalities; Fixed Point; Hierarchical Circularly Iterative Sequence; Strongly Quasi-Nonexpansive Mapping

\section{Introduction}

For a given nonlinear operator $T: H \rightarrow H$, the following classical variational inequality problem is formulated as finding a point $p^{*} \in D$ such that

$$
\left\langle T p^{*}, x-p^{*}\right\rangle \geq 0, \quad x \in D .
$$

Variational inequalities were initially studied by Stampacchia [1] and ever since have been widely studied, since they cover as diverse disciplines as partial differential equations, optimal control, optimization, mathematical programming, mechanics, and finance. On the other hand, a number of mathematical programs and iterative algorithms have been developed to resolve complex real world problems.

The concept of variational inequalities plays an important role in structural analysis, mechanics and economics. Recently, the hierarchical variational inequalities and hierarchical iterative sequence problems have attached many authors' attention (see [2-11]).

\section{Preliminaries and Lemma}

It is well-known that, for any $x \in H$, there exists a unique nearest point in $C$, denoted by $P_{C}(x)$, such that

$$
P_{C}(x)=\inf _{y \in H}\|x-y\|, \forall x \in H .
$$

Such a mapping $P_{C}$ from $H$ onto $C$ is called the metric projection.
Lemma 2.1 (see [12]) The metric projection $P_{C}: H \rightarrow C$ has the following basic properties:

1) $P_{C}$ is firmly nonexpansive, i.e.,

$$
\begin{aligned}
& \left\langle P_{C}(x)-P_{C}(y), x-y\right\rangle \\
& \geq\left\|P_{C}(x)-P_{C}(y)\right\|^{2}(\forall x, y \in H),
\end{aligned}
$$

and so $P_{C}$ is nonexpansive.

2) $\left\langle x-P_{C} x, y-P_{C} x\right\rangle \leq 0$, for all $x \in H$ and $y \in C$.

Definition 2.2

1) A mapping $T: H \rightarrow H$ is said to be $\alpha$-inversestrongly monotone if there exists $\alpha>0$ such that

$$
\langle T x-T y, x-y\rangle \geq \alpha\|T x-T y\|, \forall x, y \in H .
$$

2) A mapping $T: H \rightarrow H$ is said to be $\alpha$-Lipschitzian if

$$
\|T x-T y\| \leq \alpha\|x-y\|, \forall x, y \in H .
$$

3) A mapping $T: H \rightarrow H$ is said to be quasinonexpansive if $\operatorname{Fix}(T) \neq \Phi$ and

$$
\|T x-p\| \leq\|x-p\|, \forall x \in H, p \in \operatorname{Fix}(T) .
$$

4) A mapping $T: H \rightarrow H$ is said to be strongly quasi-nonexpansive if $T$ is quasi-nonexpansive and $x_{n}-T x_{n} \rightarrow 0$, whenever $\left\{x_{n}\right\}$ is a bounded sequence in $H$ and $\left\|x_{n}-p\right\|-\left\|T x_{n}-p\right\| \rightarrow 0$ for some $p \in \operatorname{Fix}(T)$.

5) (see [13]) A mapping $T: H \rightarrow H$ is said to be 
$\omega$-demicontractive if $\operatorname{Fix}(T) \neq \Phi$ and

$$
\langle x-T x, x-p\rangle \geq \frac{1-\omega}{2}\|x-T x\|^{2}, \forall x \in H p \in \operatorname{Fix}(T) .
$$

Obviously, the above inequality is equivalent to

$$
\|T x-p\|^{2} \leq\|x-p\|^{2}+\omega\|x-T x\|^{2},
$$

and it is clear from the preceding definitions that every quasi-nonexpansive mapping is 0-demicontractive.

Lemma 2.3 (see [14]) For $x, y \in H$ and $\omega \in[0,1]$, we have the following statements:

a) $|\langle x, y\rangle| \leq\|x\|\|y\|$

b) $\|x+y\| \leq\|x\|^{2}+2\langle y, y+x\rangle$;

c) $\|(1-\omega) x+\omega y\|^{2}$

$$
=(1-\omega)\|x\|^{2}+\omega\|y\|^{2}-\omega(1-\omega)\|x-y\|^{2} .
$$

For prove our result, we give the following lemma.

Lemma 2.4 ([11]) Let $\left\{\alpha_{n}\right\}$ be a sequence of real numbers such that there exists a subsequence $\left\{n_{i}\right\}$ of $\{n\}$ such that $\alpha_{n_{i}} \leq \alpha_{n_{i}+1}$ for all $i \in N$. Then there exists a nondecreasing $\left\{m_{k}\right\} \subset N$, such that $m_{k} \rightarrow \infty$ and the following properties are satisfied for all (sufficiently large) numbers sequence $k \subset N$ :

$$
\alpha_{m_{k}} \leq \alpha_{m_{k}+1} \text { and } \alpha_{k} \leq \alpha_{m_{k}+1} \text {. }
$$

In fact, $m_{k}=\max \left\{j \leq k: \alpha_{j} \leq \alpha_{j+1}\right\}$.

Lemma 2.5 ([11]) Assume that $\left\{\alpha_{n}\right\}$ is a sequence of nonnegative real numbers such that

$$
\alpha_{n+1} \leq\left(1-\gamma_{n}\right) \alpha_{n}+\gamma_{n} \delta_{n},
$$

where $\left\{\gamma_{n}\right\}$ is a sequence in $(0,1)$ and $\left\{\delta_{n}\right\}$ is a sequence such that (a) $\lim _{n \rightarrow \infty} \gamma_{n}=0, \sum_{n=1}^{\infty} \gamma_{n}=\infty,(b)$ $\limsup _{n \rightarrow \infty} \delta_{n} \leq 0$. Then $\lim _{n \rightarrow \infty} \alpha_{n}=0$.

Lemma 2.6 ([11]) Let $\left\{a_{n}\right\} \subset[0, \infty)$,

$$
\left\{\alpha_{n}\right\} \subset[0,1],\left\{b_{n}\right\} \subset(-\infty,+\infty)
$$

and $\lambda \in[0,1]$, such that

- $\left\{a_{n}\right\}$ is a bounded sequence;

- $a_{n+1} \leq\left(1-\alpha_{n}\right)^{2} a_{n}+2 \alpha_{n} \lambda \sqrt{a_{n}} \sqrt{a_{n+1}}+\alpha_{n} b_{n}$, for all $n \in N$;

- whenever $\left\{a_{n_{k}}\right\}$ is a subsequence of $\left\{a_{n}\right\}$ satisfying $\liminf _{k \rightarrow \infty}\left(a_{n_{k}+1}-a_{n_{k}}\right) \geq 0$, it follows that

$$
\limsup _{k \rightarrow \infty} b_{n_{k}} \leq 0 \text {; }
$$

- $\lim _{n \rightarrow \infty} \alpha_{n}=0, \sum_{n=1}^{\infty} \alpha_{n}=\infty$.

Then $\lim _{n \rightarrow \infty} a_{n}=0$.

In [11], the existence and uniqueness of solutions of some related hierarchical optimization problems had been discussed.

Inspired by these results in the literature, a circularly iterative method in this paper is introduced for solving a system of variational inequalities with fixed-point set constraints. Under suitable conditions, strong convergence results are proved in the setting of Hilbert spaces. Our scheme can be regarded as a more general variant of the algorithm proposed by Maingé. The results presented in the paper improve and extend the corresponding results in [11] and other.

\section{Main Results}

First, we discuss the existence and uniqueness of solutions of some related hierarchical optimization problems.

Theorem 3.1 Let $S_{i}: H \rightarrow H$ be quasi-nonexpansive mappings and $f_{i}: H \rightarrow H$ be contractions $(i=1,2, \cdots, m)$. Then there exists a unique element $p_{i} \in \operatorname{Fix}\left(S_{i}\right)$ such that the following inequalities,

$$
\left\{\begin{array}{l}
\left\langle p_{i}-f_{i}\left(p_{i+1}\right), u_{i}-p_{i}\right\rangle \geq 0, \\
\forall u_{i} \in \operatorname{Fix}\left(S_{i}\right), i=1,2, \cdots, m-1, \\
\left\langle p_{m}-f_{m}\left(p_{1}\right), u_{m}-p_{m}\right\rangle \geq 0, \\
\forall u_{m} \in \operatorname{Fix}\left(S_{m}\right) .
\end{array}\right.
$$

Proof. The proof is a consequence of the well-known Banach's contraction principle but it is given here for the sake of completeness. It is known that both sets Fix $\left(S_{i}\right)(i=1,2, \cdots, m)$ are closed and convex, and hence the projections $P_{F i x\left(S_{i}\right)}(i=1,2, \cdots, m)$ are well defined. It is clear that the mapping

$$
P_{F i x\left(S_{1}\right)} \cdot f_{1} \cdot P_{F i x\left(S_{2}\right)} \cdot f_{2} \cdots P_{F i x\left(S_{m}\right)} \cdot f_{m}
$$

is a contraction. Hence, there exists a unique element $p_{1} \in H$ such that

$$
p_{1}=\left(P_{F i x\left(S_{1}\right)} \cdot f_{1} \cdot P_{F i x\left(S_{2}\right)} \cdot f_{2} \cdots P_{F i x\left(S_{m}\right)} \cdot f_{m}\right) p_{1} .
$$

Put $p_{m}=P_{F i x\left(S_{m}\right)} f_{m} p_{1}$ and

$$
p_{i}=P_{F i x\left(S_{1}\right)} f_{1} p_{i+1}(i=1,2, \cdots, m-1) .
$$

Then $p_{2} \in P_{F i x\left(S_{2}\right)}, \quad p_{3} \in P_{F i x\left(S_{3}\right)}$ and $p_{1}=P_{F i x\left(S_{1}\right)} f_{1} p_{2}$. Suppose that there is an element

$$
p_{i}^{*} \in \operatorname{Fix}\left(S_{i}\right)(i=1,2, \cdots, m),
$$

such that the following inequalities,

$$
\left\{\begin{array}{l}
\left\langle p_{i}^{*}-f_{i}\left(p_{i+1}^{*}\right), u_{i}-p_{i}^{*}\right\rangle \geq 0, \\
\forall u_{i} \in \operatorname{Fix}\left(S_{i}\right), i=1,2, \cdots, m-1, \\
\left\langle p_{m}^{*}-f_{m}\left(p_{1}^{*}\right), u_{m}-p_{m}^{*}\right\rangle \geq 0, \\
\forall u_{m} \in \operatorname{Fix}\left(S_{m}\right) .
\end{array}\right.
$$

Then $p_{m}^{*}=P_{F i x\left(S_{m}\right)} f_{m} p_{1}^{*}$ and

$$
p_{i}^{*}=P_{F i x\left(S_{1}\right)} f_{1} p_{i+1}^{*}(i=1,2, \cdots, m-1) \text {. }
$$


Hence,

$$
p_{1}^{*}=\left(P_{F i x\left(S_{1}\right)} \cdot f_{1} \cdot P_{F i x\left(S_{2}\right)} \cdot f_{2} \cdots P_{F i x\left(S_{m}\right)} \cdot f_{m}\right) p_{1}^{*} .
$$

This implies that $p_{1}=p_{1}^{*}$ and hence

$$
p_{2}=p_{2}^{*}, \cdots, p_{m}=p_{m}^{*} .
$$

This completes the proof.

For mappings $S_{i}, f_{i}: H \rightarrow H$, suppose $i=1,2, \cdots, m$, we define the iterative sequences $\left\{x_{n}^{(i)}\right\}$ by

$$
\left\{\begin{array}{l}
x_{0}^{(i)} \in H \\
x_{n+1}^{(1)}=\left(1-\alpha_{n}\right) S_{1} x_{n}^{(1)}+\alpha_{n} f_{1}\left(S_{2} x_{n}^{(2)}\right), \\
x_{n+1}^{(2)}=\left(1-\alpha_{n}\right) S_{2} x_{n}^{(2)}+\alpha_{n} f_{2}\left(S_{3} x_{n}^{(3)}\right) \\
\quad \vdots \\
x_{n+1}^{(m-1)}=\left(1-\alpha_{n}\right) S_{m-1} x_{n}^{(m-1)}+\alpha_{n} f_{m-1}\left(S_{m} x_{n}^{(m)}\right), \\
x_{n+1}^{(m)}=\left(1-\alpha_{n}\right) S_{m} x_{n}^{(m)}+\alpha_{n} f_{m}\left(S_{1} x_{n}^{(1)}\right)
\end{array}\right.
$$

$$
\begin{aligned}
\left\|x_{n+1}^{(1)}-p_{1}\right\| & \leq\left(1-\alpha_{n}\right)\left\|S_{1} x_{n}^{(1)}-p_{1}\right\|+\alpha_{n}\left\|f_{1}\left(S_{2} x_{n}^{(2)}\right)-p_{1}\right\| \\
& \leq\left(1-\alpha_{n}\right)\left\|x_{n}^{(1)}-p_{1}\right\|+\alpha_{n}\left\|f_{1}\left(S_{2} x_{n}^{((2))}\right)-f_{1}\left(p_{2}\right)\right\|+\alpha_{n}\left\|f_{1}\left(p_{2}\right)-p_{1}\right\| \\
& \leq\left(1-\alpha_{n}\right)\left\|x_{n}^{(1)}-p_{1}\right\|+\alpha_{n} \hat{\alpha}\left\|S_{2} x_{n}^{(2)}-p_{2}\right\|+\alpha_{n}\left\|f_{1}\left(p_{2}\right)-p_{1}\right\| \\
& \leq\left(1-\alpha_{n}\right)\left\|x_{n}^{(1)}-p_{1}\right\|+\alpha_{n} \hat{\alpha}\left\|x_{n}^{(2)}-p_{2}\right\|+\alpha_{n}\left\|f_{1}\left(p_{2}\right)-p_{1}\right\| .
\end{aligned}
$$

Similarly, we also have

$$
\left\{\begin{array}{c}
\left\|x_{n+1}^{(2)}-p_{2}\right\| \leq\left(1-\alpha_{n}\right)\left\|x_{n}^{(2)}-p_{2}\right\|+\alpha_{n} \hat{\alpha}\left\|x_{n}^{(3)}-p_{3}\right\|+\alpha_{n}\left\|f_{2}\left(p_{3}\right)-p_{2}\right\|, \\
\vdots \\
\left\|x_{n+1}^{(m-1)}-p_{m-1}\right\| \leq\left(1-\alpha_{n}\right)\left\|x_{n}^{(m-1)}-p_{m-1}\right\|+\alpha_{n} \hat{\alpha}\left\|x_{n}^{(m)}-p_{m}\right\|+\alpha_{n}\left\|f_{m-1}\left(p_{m}\right)-p_{m-1}\right\|, \\
\left\|x_{n+1}^{(m)}-p_{m}\right\| \leq\left(1-\alpha_{n}\right)\left\|x_{n}^{(m)}-p_{m}\right\|+\alpha_{n} \hat{\alpha}\left\|x_{n}^{(1)}-p_{1}\right\|+\alpha_{n}\left\|f_{m}\left(p_{1}\right)-p_{m}\right\| .
\end{array}\right.
$$

It implies that

$$
\begin{aligned}
& \sum_{i=1}^{m}\left\|x_{n+1}^{(i)}-p_{i}\right\| \leq\left[1-(1-\hat{\alpha}) \alpha_{n}\right] \sum_{i=1}^{m}\left\|x_{n}^{(i)}-p_{i}\right\| \\
& +\alpha_{n}\left(\left\|f_{1}\left(p_{2}\right)-p_{1}\right\|+\left\|f_{2}\left(p_{3}\right)-p_{2}\right\|+\cdots+\left\|f_{m-1}\left(p_{m}\right)-p_{m-1}\right\|+\left\|f_{m}\left(p_{1}\right)-p_{m}\right\|\right) \\
& \leq \max \left\{\sum_{i=1}^{m}\left\|x_{n}^{(i)}-p_{i}\right\|, \frac{\left\|f_{1}\left(p_{2}\right)-p_{1}\right\|+\left\|f_{2}\left(p_{3}\right)-p_{2}\right\|+\cdots+\left\|f_{m-1}\left(p_{m}\right)-p_{m-1}\right\|+\left\|f_{m}\left(p_{1}\right)-p_{m}\right\|}{1-\hat{\alpha}}\right\} .
\end{aligned}
$$

By induction, we have

$$
\sum_{i=1}^{m}\left\|x_{n+1}^{(i)}-p_{i}\right\| \leq \max \left\{\sum_{i=1}^{m}\left\|x_{0}^{(i)}-p_{i}\right\|, \frac{\left\|f_{1}\left(p_{2}\right)-p_{1}\right\|+\left\|f_{2}\left(p_{3}\right)-p_{2}\right\|+\cdots+\left\|f_{m-1}\left(p_{m}\right)-p_{m-1}\right\|+\left\|f_{m}\left(p_{1}\right)-p_{m}\right\|}{1-\hat{\alpha}}\right\},
$$


618

H. B. DIU

for all $n \in N$. In particular, sequences $\left\{x_{n}^{(i)}\right\}$ are bounded. Consequently, the sequences $\left\{S_{i} x_{n}^{(i)}\right\}$ are also bounded.

Lemma 3.4 For each $n \in N$, the following inequality holds:

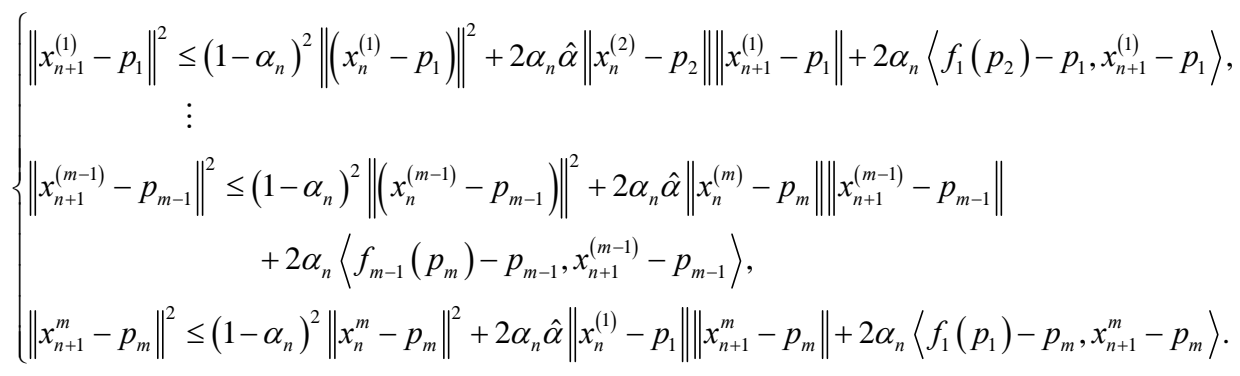

Proof. Since

$$
\begin{aligned}
& \left\|x_{n+1}^{(1)}-p_{1}\right\|^{2}=\left\|\left(1-\alpha_{n}\right)\left(S_{1} x_{n}^{(1)}-p_{1}\right)+\alpha_{n}\left(f_{1}\left(S_{2} x_{n}^{(2)}\right)-p_{1}\right)\right\|^{2} \\
& \leq\left\|\left(1-\alpha_{n}\right)\left(S_{1} x_{n}^{(1)}-p_{1}\right)\right\|^{2}+2\left\langle\alpha_{n}\left(f_{1}\left(S_{2} x_{n}^{(2)}\right)-p_{1}\right), x_{n+1}^{(1)}-p_{1}\right\rangle \\
& \leq\left(1-\alpha_{n}\right)^{2}\left\|\left(S_{1} x_{n}^{(1)}-p_{1}\right)\right\|^{2}+2 \alpha_{n}\left\langle f_{1}\left(S_{2} x_{n}^{(2)}\right)-f_{1}\left(p_{2}\right), x_{n+1}^{(1)}-p_{1}\right\rangle+2 \alpha_{n}\left\langle f_{1}\left(p_{2}\right)-p_{1}, x_{n+1}^{(1)}-p_{1}\right\rangle \\
& \leq\left(1-\alpha_{n}\right)^{2}\left\|\left(S_{1} x_{n}^{(1)}-p_{1}\right)\right\|^{2}+2 \alpha_{n}\left\|f_{1}\left(S_{2} x_{n}^{(2)}\right)-f_{1}\left(p_{2}\right)\right\|\left\|x_{n+1}^{(1)}-p_{1}\right\| \\
& \leq\left(1-\alpha_{n}\right)^{2}\left\|\left(x_{n}^{(1)}-p_{1}\right)\right\|^{2}+2 \alpha_{n} \hat{\alpha}\left\|S_{2} x_{n}^{(2)}-p_{2}\right\|\left\|x_{n+1}^{(1)}-p_{1}\right\| \\
& \quad+2 \alpha_{n}\left\langle f_{1}\left(p_{2}\right)-p_{1}, x_{n+1}^{(1)}-p_{1}\right\rangle+2 \alpha_{n}\left\langle f_{1}\left(p_{2}\right)-p_{1}, x_{n+1}^{(1)}-p_{1}\right\rangle \\
& \leq\left(1-\alpha_{n}\right)^{2}\left\|\left(x_{n}^{(1)}-p_{1}\right)\right\|^{2}+2 \alpha_{n} \hat{\alpha}\left\|x_{n}^{(2)}-p_{2}\right\|\left\|x_{n+1}^{(1)}-p_{1}\right\|+2 \alpha_{n}\left\langle f_{1}\left(p_{2}\right)-p_{1}, x_{n+1}^{(1)}-p_{1}\right\rangle .
\end{aligned}
$$

Similarly, we also have

$$
\left\{\begin{array}{c}
\left\|x_{n+1}^{(2)}-p_{2}\right\|^{2} \leq\left(1-\alpha_{n}\right)^{2}\left\|\left(x_{n}^{(2)}-p_{2}\right)\right\|^{2}+2 \alpha_{n} \hat{\alpha}\left\|x_{n}^{(3)}-p_{3}\right\|\left\|x_{n+1}^{(2)}-p_{2}\right\|+2 \alpha_{n}\left\langle f_{2}\left(p_{3}\right)-p_{2}, x_{n+1}^{(2)}-p_{2}\right\rangle, \\
\begin{array}{rl}
\left\|x_{n+1}^{(m-1)}-p_{m-1}\right\|^{2} \leq & \left(1-\alpha_{n}\right)^{2}\left\|\left(x_{n}^{(m-1)}-p_{m-1}\right)\right\|^{2}+2 \alpha_{n} \hat{\alpha}\left\|x_{n}^{(m)}-p_{m}\right\|\left\|x_{n+1}^{(m-1)}-p_{m-1}\right\| \\
& +2 \alpha_{n}\left\langle f_{m-1}\left(p_{m}\right)-p_{m-1}, x_{n+1}^{(m-1)}-p_{m-1}\right\rangle,
\end{array} \\
\left\|x_{n+1}^{m}-p_{m}\right\|^{2} \leq\left(1-\alpha_{n}\right)^{2}\left\|x_{n}^{m}-p_{m}\right\|^{2}+2 \alpha_{n} \hat{\alpha}\left\|x_{n}^{(1)}-p_{1}\right\|\left\|x_{n+1}^{m}-p_{m}\right\|+2 \alpha_{n}\left\langle f_{1}\left(p_{1}\right)-p_{m}, x_{n+1}^{m}-p_{m}\right\rangle .
\end{array}\right.
$$

By Lemma 3.3, we give following result,

$$
\begin{aligned}
& \left\|x_{n+1}^{(1)}-p_{1}\right\|^{2}+\left\|x_{n+1}^{(2)}-p_{2}\right\|^{2}+\cdots+\left\|x_{n+1}^{(m)}-p_{m}\right\|^{2} \leq\left(1-\alpha_{n}\right)^{2}\left(\left\|\left(x_{n}^{(1)}-p_{1}\right)\right\|^{2}+\left\|\left(x_{n}^{(2)}-p_{2}\right)\right\|^{2}+\cdots+\left\|\left(x^{(m)}-p_{m}\right)\right\|^{2}\right) \\
& +2 \alpha_{n} \hat{\alpha}\left(\left\|x_{n}^{(2)}-p_{2}\right\|\left\|x_{n+1}^{(1)}-p_{1}\right\|+\left\|x_{n}^{(2)}-p_{2}\right\|\left\|x_{n+1}^{(2)}-p_{2}\right\|+\cdots+\left\|x_{n}^{(1)}-p_{1}\right\|\left\|x_{n+1}^{(m)}-p_{m}\right\|\right) \\
& +2 \alpha_{n}\left(\left\langle f_{1}\left(p_{2}\right)-p_{1}, x_{n+1}^{(1)}-p_{1}\right\rangle+\left\langle f_{2}\left(p_{3}\right)-p_{2}, x_{n+1}^{(2)}-p_{2}\right\rangle+\cdots+\left\langle f_{m}\left(p_{1}\right)-p_{m}, x_{n+1}^{(m)}-p_{m}\right\rangle\right) .
\end{aligned}
$$

Lemma 3.5 If there exists a subsequence $\left\{n_{k}\right\}$ of $\{n\}$ such that

Copyright (C) 2013 SciRes.

AP 


$$
\liminf _{k \rightarrow \infty}\left(\sum_{i=1}^{m}\left\|x_{n_{k}+1}^{(i)}-p_{i}\right\|^{2}-\sum_{i=1}^{m}\left\|x_{n_{k}}^{(i)}-p_{i}\right\|^{2}\right) \geq 0,
$$

then

$$
\underset{k \rightarrow \infty}{\limsup }\left(\sum_{i=1}^{m-1}\left\langle f_{i}\left(p_{i+1}\right)-p_{i}, x_{n_{k}+1}^{(i)}-p_{i}\right\rangle+\left\langle f_{m}\left(p_{1}\right)-p_{m}, x_{n_{k}+1}^{(m)}-p_{m}\right\rangle\right) \leq 0
$$

Proof. In fact, we first consider the following assertion:

$$
\begin{aligned}
0 & \leq \liminf _{k \rightarrow \infty}\left(\sum_{i=1}^{m}\left\|x_{n_{k}+1}^{(i)}-p_{i}\right\|^{2}-\sum_{i=1}^{m}\left\|x_{n_{k}}^{(i)}-p_{i}\right\|^{2}\right) \leq \liminf _{k \rightarrow \infty}\left[\sum_{i=1}^{m-1}\left(1-\alpha_{n_{k}}\right)\left\|S_{i} x_{n_{k}}^{(i)}-p_{i}\right\|^{2}+\alpha_{n_{k}}\left\|f_{i}\left(S_{i+1} x_{n_{k}}^{(i+1)}\right)-p_{i+1}\right\|^{2}\right. \\
& \left.+\left(1-\alpha_{n_{k}}\right)\left\|S_{m} x_{n_{k}}^{(m)}-p_{m}\right\|^{2}+\alpha_{n_{k}}\left\|f_{m}\left(S_{1} x_{n_{k}}^{(m)}\right)-p_{1}\right\|^{2}-\sum_{i=1}^{m}\left\|x_{n_{k}}^{(i)}-p_{i}\right\|^{2}\right] \\
& =\liminf _{k \rightarrow \infty} \sum_{i=1}^{m}\left(\left\|S_{i} x_{n_{k}}^{(i)}-p_{i}\right\|^{2}-\left\|x_{n_{k}}^{(i)}-p_{i}\right\|^{2}\right) \leq \limsup _{k \rightarrow \infty} \sum_{i=1}^{m}\left(\left\|S_{i} x_{n_{k}}^{(i)}-p_{i}\right\|^{2}-\left\|x_{n_{k}}^{(i)}-p_{i}\right\|^{2}\right) \leq 0 .
\end{aligned}
$$

By Lemma 3.3, the sequences

$$
\left\{\left\|S_{1} x_{n_{k}}^{(i)}-p_{i}\right\|+\left\|x_{n_{k}}^{(i)}-p\right\|\right\}(i=1,2, \cdots, m)
$$

are bounded. So we have

$$
\begin{aligned}
& \lim _{k \rightarrow \infty}\left(\left\|S_{i} x_{n_{k}}^{(i)}-p_{i}\right\|^{2}-\left\|x_{n_{k}}^{(i)}-p_{i}\right\|^{2}\right)=0, \\
& (i=1,2, \cdots, m)
\end{aligned}
$$

Since $S_{i}(i=1,2,3)$ are strongly quasi-nonexpansive,

$$
\lim _{k \rightarrow \infty}\left(S_{i} x_{n_{k}}^{(i)}-x_{n_{k}}^{(i)}\right)=0,(i=1,2, \cdots, m) .
$$

by the iteration scheme (1), we have

$$
\lim _{k \rightarrow \infty}\left(x_{n_{k}}^{(i)}-x_{n_{k}+1}^{(i)}\right)=0,(i=1,2, \cdots, m) .
$$

It follows from the boundedness of $\left\{x_{n_{k}}^{(1)}\right\}$ that there exists a subsequence $\left\{x_{n_{k_{l}}}^{(1)}\right\}$ of $\left\{x_{n_{k}}^{(1)}\right\}$ such that $\left\{x_{n_{k l}}^{(1)}\right\} \rightarrow x$ and

$$
\begin{aligned}
& \lim _{l \rightarrow \infty}\left\langle f_{1}\left(p_{2}\right)-p_{1}, x_{n_{k_{l}}}^{(1)}-p_{1}\right\rangle \\
& =\limsup _{k \rightarrow \infty}\left\langle f_{1}\left(p_{2}\right)-p_{1}, x_{n_{k}}^{(1)}-p_{1}\right\rangle \\
& =\limsup _{k \rightarrow \infty}\left\langle f_{1}\left(p_{2}\right)-p_{1}, x_{n_{k}+1}^{(1)}-p_{1}\right\rangle .
\end{aligned}
$$

Since $I-S_{1}$ is demiclosed at zero, it follows that $x \in \operatorname{Fix}\left(S_{1}\right)$. It follows from (1), we get

$$
\begin{aligned}
& \lim _{l \rightarrow \infty}\left\langle f_{1}\left(p_{2}\right)-p_{1}, x_{n_{k_{l}}}^{(1)}-p_{1}\right\rangle \\
& =\left\langle f_{1}\left(p_{2}\right)-p_{1}, x-p_{1}\right\rangle \leq 0 .
\end{aligned}
$$

Consequently,

$$
\limsup \sup _{k \rightarrow \infty}\left\langle f_{1}\left(p_{2}\right)-p_{1}, x_{n_{k}+1}^{(1)}-p_{1}\right\rangle \leq 0 .
$$

By using the same argument, we have

$$
\left\{\begin{array}{c}
\limsup _{k \rightarrow \infty}\left\langle f_{2}\left(p_{3}\right)-p_{2}, x_{n_{k}+1}^{(2)}-p_{2}\right\rangle \leq 0, \\
\limsup _{k \rightarrow \infty}\left\langle f_{3}\left(p_{4}\right)-p_{3}, x_{n_{k}+1}^{(3)}-p_{3}\right\rangle \leq 0, \\
\vdots \\
\limsup _{k \rightarrow \infty}\left\langle f_{m}\left(p_{1}\right)-p_{m}, x_{n_{k}+1}^{(m)}-p_{m}\right\rangle \leq 0 .
\end{array}\right.
$$

Therefore, we obtain the desired inequality (4). Next, we prove Theorem 3.2. Denote

$$
\begin{aligned}
& a_{n}:=\sum_{i=1}^{m}\left\|x_{n}^{(i)}-p_{i}\right\|^{2} \\
& b_{n}:=2\left(\left\langle f_{1}\left(p_{2}\right)-p_{1}, x_{n+1}^{(1)}-p_{1}\right\rangle\right. \\
& +\left\langle f_{2}\left(p_{3}\right)-p_{2}, x_{n+1}^{(2)}-p_{2}\right\rangle+\cdots \\
& \left.+\left\langle f_{m}\left(p_{1}\right)-p_{m}, x_{n+1}^{(m)}-p_{m}\right\rangle\right) .
\end{aligned}
$$

Since

$$
\begin{aligned}
& \left\|x_{n}^{(2)}-p_{2}\right\|\left\|x_{n+1}^{(1)}-p_{1}\right\|+\left\|x_{n}^{(3)}-p_{3}\right\|\left\|x_{n+1}^{(2)}-p_{2}\right\|+\cdots \\
& +\left\|x_{n}^{(1)}-p_{1}\right\|\left\|x_{n+1}^{(m)}-p_{m}\right\| \\
& \leq\left(\sum_{i=1}^{m}\left\|x_{n}^{(i)}-p_{i}\right\|^{2}\right)^{\frac{1}{2}} \times\left(\sum_{i=1}^{m}\left\|x_{n+1}^{(i)}-p_{i}\right\|^{2}\right)^{\frac{1}{2}},
\end{aligned}
$$

We have the following statements from Lemma (3.3), Lemma(3.4) and Lemma(3.5):

- $\left\{a_{n}\right\}$ is a bounded sequence;

- $a_{n+1} \leq\left(1-\alpha_{n}\right)^{2} a_{n}+2 \alpha_{n} \lambda \sqrt{a_{n}} \sqrt{a_{n+1}}+\alpha_{n} b_{n}$, for all $n \in N$;

- whenever $\left\{a_{n_{k}}\right\}$ is a subsequence of $\left\{a_{n}\right\}$ satisfying $\liminf _{k \rightarrow \infty}\left(a_{n_{k}+1}-a_{n_{k}}\right) \geq 0$, it follows that 
$\limsup _{k \rightarrow \infty} b_{n_{k}} \leq 0$.

Hence, it follows from Lemma 2.6 that $a_{n} \rightarrow 0$, It implies that

$$
\lim _{n \rightarrow \infty} \sum_{i=1}^{m}\left\|x_{n}^{(i)}-p_{i}\right\|^{2}=0 .
$$

This means that

$$
\lim _{n \rightarrow \infty} x^{(i)}=p_{i}(i=1,2, \cdots, m) .
$$

The proof of Theorem 3.2 is completed.

\section{REFERENCES}

[1] D. Kinderlehrer and G. Stampacchia, “An Introduction to Variational Inequalities and Their Applications,” Classics in Applied Mathematics, Vol. 31, SIAM, Philadelphia, 2000. http://dx.doi.org/10.1137/1.9780898719451

[2] E. Buzogány, I. Mezei and V. Varga, "Two-Variable Variational-Hemivariational Inequalities," Studia Universitatis Babeş-Bolyai Mathematica, Vol. 47, No. 3, 2002, pp. 31-41.

[3] E. Blum and W. Oettli, "From Optimization and Variational Inequalities to Equilibrium Problems,” Mathematics Student-India, Vol. 63, No. 1, 1994, pp. 123-145.

[4] R. U. Verma, "Projection Methods, Algorithms and A New System of Nonlinear Variational Inequalities," Computers \& Mathematics with Applications, Vol. 41, No. 7-8, 2001, pp. 1025-1031. http://dx.doi.org/10.1016/S0898-1221(00)00336-9

[5] D. Kinderlehrer and G. Stampacchia, "An Introduction to Variational Inequalities and Their Applications,” Academic Press, New York, 1980.

[6] M. A. Noor and K. I. Noor, "Sensitivity Analysis of Quasi Variational Inclusions,” Journal of Mathematical
Analysis and Applications, Vol. 236, No. 2, 1999, pp. 290-299. http://dx.doi.org/10.1006/jmaa.1999.6424

[7] S. S. Chang, "Set-Valued Variational Inclusions in Banach Spaces," Journal of Mathematical Analysis and Applications, Vol. 248, No. 2, 2000, pp. 438-454. http://dx.doi.org/10.1006/jmaa.2000.6919

[8] S. S. Chang, "Existence and Approximation of Solutions of Set-Valued Variational Inclusions in Banach Spaces," Nonlinear Analysis, Vol. 47, No. 1, 2001, pp. 583-494. http://dx.doi.org/10.1016/S0362-546X(01)00203-6

[9] V. F. Demyanov, G. E. Stavroulakis, L. N. Polyakova and P. D. Panagiotopoulos, "Quasidifferentiability and Nonsmooth Modeling in Mechanics, Engineering and Economics,” Kluwer Academic, Dordrecht, 1996.

[10] P. E. Maingé and A. Mouda, "Strong Convergence of an Iterative Method for Hierarchical Fixed Point Problems," Pacific Journal of Optimization, Vol. 3, No. 3, 2007, pp. 529-538.

[11] R. Kraikaew and S. Saejung, “On Maingé’s Approach for Hierarchical Optimization Problems,” Journal of Optimization Theory and Applications, Vol. 154, No. 1, 2012, pp. 71-87. http://dx.doi.org/10.1007/s10957-011-9982-4

[12] S. S. Zhang, Joseph H. W. Lee and C. K. Chan, “Algorithms of Common Solutions for Quasi Variational Inclusion and Fixed Point Problems," Applied Mathematics and Mechanics, Vol. 29, No. 5, 2008, pp. 1-11. http://dx.doi.org/10.1007/s10483-008-0101-7

[13] T. L. Hicks and J. D. Kubicek, "On the Mann Iteration Process in a Hilbert Space,” Journal of Mathematical Analysis and Applications, Vol. 59, No. 3, 1977, pp. 498504. http://dx.doi.org/10.1016/0022-247X(77)90076-2

[14] W. Takahashi, "Introduction to Nonlinear and Convex Analysis,” Yokohama Publishers, Yokohama, 2009. 